\title{
Implementing New LTE GUI Dimensioning Tool
}

\author{
Eman S. El-Din and Ibraheem M. Fayed \\ Network Planning Department, National Telecommunication Institute (NTI), Cairo, Egypt
}

\begin{abstract}
The research area of mobile network has increased rapidly due to the new technologies in this field. In the RNP (radio network planning), the dimensioning of LTE (long term evolution) radio access network is a very important and critical process to get the required network performance and optimal cost. The planning and dimensioning simulation tools play an important role to select the optimal parameters values. A simulation dimensioning tool is introduced with GUI using MATLAB programs to represent mathematical formulas of LTE planning. The purpose of designing new tool is to be aware of the concept of planning, steps, different parameters and calculations used to understand planning better. A dimensioning tool that makes use of the link budget for cell coverage should be used, where a system level simulator may be used for the best estimation of capacity which is an input for the tool. The tool determines the cell range using coverage analysis. Then a capacity analysis is performed for capacity requirements. At the end the cell radius, throughput and the number of sites are determined. In this paper, a detailed LTE radio network planning procedure has been clearly presented which concentrates on coverage and capacity estimations. The tool is capable of dimensioning LTE network to give the number of sites of the base stations needed to cover a certain area. The simulator results are verified by other simulation tool (ATOLL simulation) which gives the same results as the new simulator tool.
\end{abstract}

Key words: Network simulation tool, LTE planning, capacity, and coverage.

\section{Introduction}

The radio network planning process is designed to maximize the network coverage while providing the desired capacity simultaneously. The network must offer sufficient coverage and capacity while maintaining the lowest deployment cost [1].

The main steps in the network planning process are: pre-planning, nominal planning, detailed planning, verification and optimization.

Dimensioning is the initial phase of network planning. It provides the first estimate of the network element count as well as the capacity of those elements. The purpose of dimensioning is to estimate the required number of radio base stations needed to support a specified traffic load in an area and the specific service to the cell edge users. Dimensioning is an important part of the whole planning process, which also includes detailed planning and optimization of the wireless cellular network. The

Corresponding author: Ibraheem M. Fayed, assistant professor, research fields: wireless networks, mobile cellular networks and communication systems. target of the LTE access network dimensioning is to estimate the required site density and site configurations for the area of interest. Initial LTE access network planning activities include radio link budget and coverage analysis, cell capacity estimation, estimation of the amount of eNB [2].

A network simulator is a methodology to implement the network on the computer. The simulation in computer can model virtual and real-life objects on a computer. Therefore, it can be studied.

The behavior of the network may be calculated either by network entities interconnection using analytical solution. Also it can be modeled by considering the history experience from a production network [3]. Wireless networks optimize devices with respect to computing power and wireless communication.

In this paper, a simulation tool using MatLab environment $[4,5]$ is introduced. This tool is based on GUI interface to study and analyze LTE planning Dimensioning with its two steps: Coverage and Capacity calculation in LTE network to give the 
number of sites of the base stations needed to cover a certain area.

This paper is organized as follows: The planning in LTE network is in Section 2; The parameters used in GUI of dimensioning simulation tool are explained in Section 3; In section 4, the simulation results for Coverage and Capacity calculation in LTE network to give the number of sites are shown and verified by certified simulation tool (ATOLL simulation) [6]; Section 5 is devoted to conclusion.

\section{LTE Planning}

The main steps in the network planning process are: pre-planning, nominal planning, detailed planning, verification and optimization. The input for the preplanning phase is the network planning criteria. The main activity is dimensioning, which gives the initial network configuration as a result. The first step in the planning phase is nominal planning; it provides the first site locations in the map based on input from the dimensioning phase. The process continues with more detailed coverage planning after site hunting and transmission planning. Detailed Capacity planning is also included in the planning phase. Detailed planning covers frequency, neighbor and parameter planning. After detailed planning the network is ready for verification and acceptance, which finishes the prelaunch activities. After the launch the activities continue with optimization as shown in Fig. 1.

Dimensioning Planning which consists the pre-planning phase is the preparation before actual network planning process starts. A detailed survey is done in order to gather information about the area to be planned, the standard of living, the rush hour and many other things that will help setting the planning network criteria. The necessary information can be as follows:

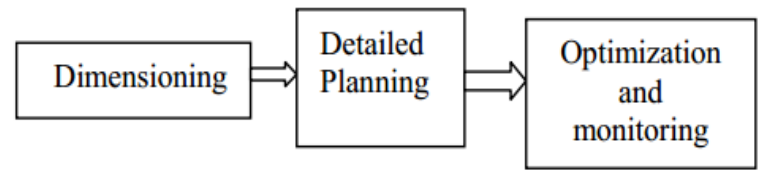

Fig. 1 Planning steps.
- User related: User density in different areas, bit rate requirements in each area, data traffic class of users, payment capacity and tolerance to unexpected quality.

- Terrain characteristics: Location and heights of buildings, foliage and highway overpasses. Also rain and other atmospheric characteristics will be useful.

- E-NodeB/UE related: Transmitted power, antenna type, antenna gain, feeder loss, body loss and other environmental losses from Ref. [7].

- Available spectrum.

- Market setup.

- Data resolution and cost of data.

Dimensioning planning determines the number of sites required to cover the target areas while fulfilling the coverage and capacity requirements.

\section{Model Parameters and Assumptions}

A MatLab code is used to simulate the number of eNB in LTE network respect to different services are types. It is used to apply the parameters of different operational frequencies and different modulation schemes to estimate coverage and capacity.

It consists of mainly 5 classes:
(a) Estimation capacity;
(b) Estimation coverage;
(c) Site inforsmation;
(d) Graph.

\subsection{Code Features}

Coverage Calculations

- Calculating the link budget parameters plus the different parameters related to the link.

- The propagation models calculations.

Capacity Calculations

- Average throughput per sub (UL and DL) calculations

- Average throughput per site (UL and DL) calculations

- The different modulation schemes.

Site Information 
- Frequency carriers, area name, population, growth rate.

Total number of sites

- According to coverage calculation.

- According to capacity calculation.

- According to coverage and capacity calculation.

- The code plot the total number of sites respects to different services area types, modulation schemes, frequencies.

\subsection{Flow Chart Presents Calculations on MATLAB}

The program includes several functions used to calculate models' equation parameters. Fig. 2 is a layout of the GUI interface.

The nature types the area to be covered as shown in Fig. 2 can be divided into three types:

(a) Urban: is characterized by higher population density, urban areas may be cities or towns;

(b) Suburban: can be the residential areas of a large city, or separate residential communities within commuting distance of a city;

(c) Rural: are large and isolated areas of an open country, often with low population density.

New simulation tool version supports link mode:

(a) DL: indicate the downlink mode;

(b) UL: indicate the uplink mode.

\section{GUI Simulation Output}

Figs. 3-7 represent the output of the GUI interface of new simulation with five selected parts.

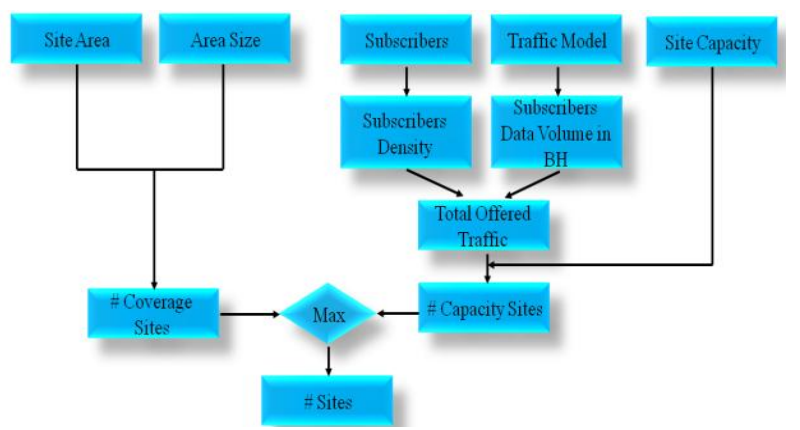

Fig. 2 MATLAB flow chart.

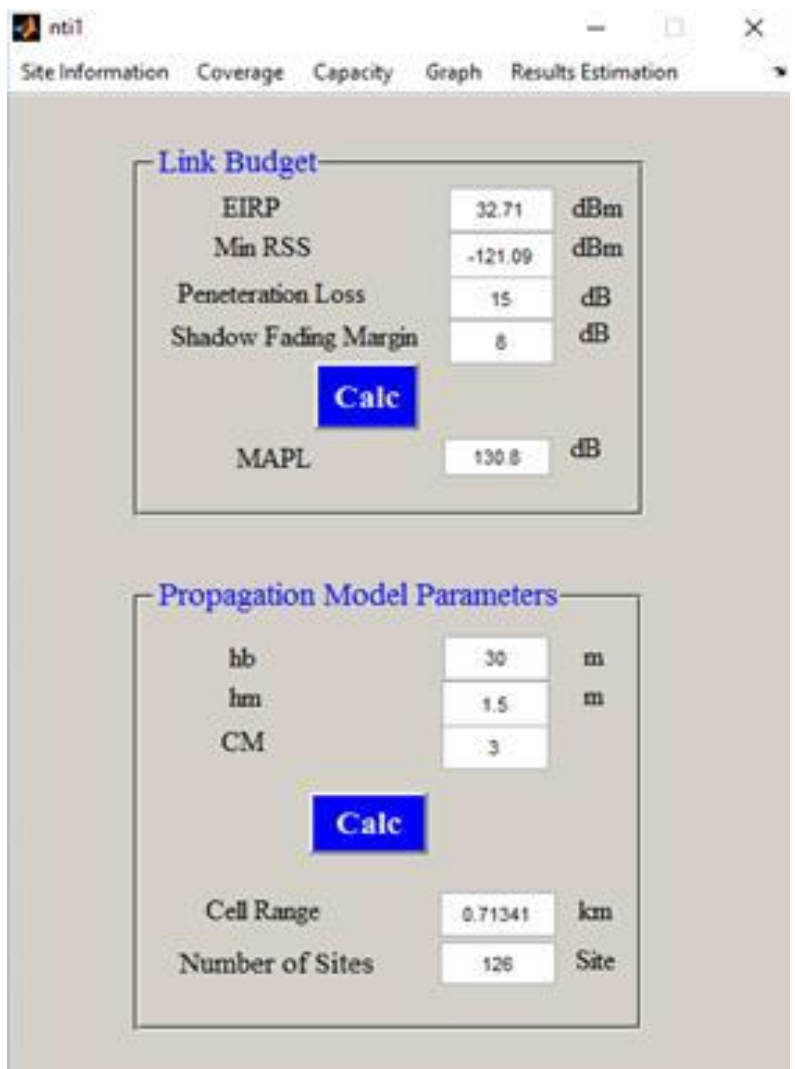

Fig. 3 GUI coverage calculations.

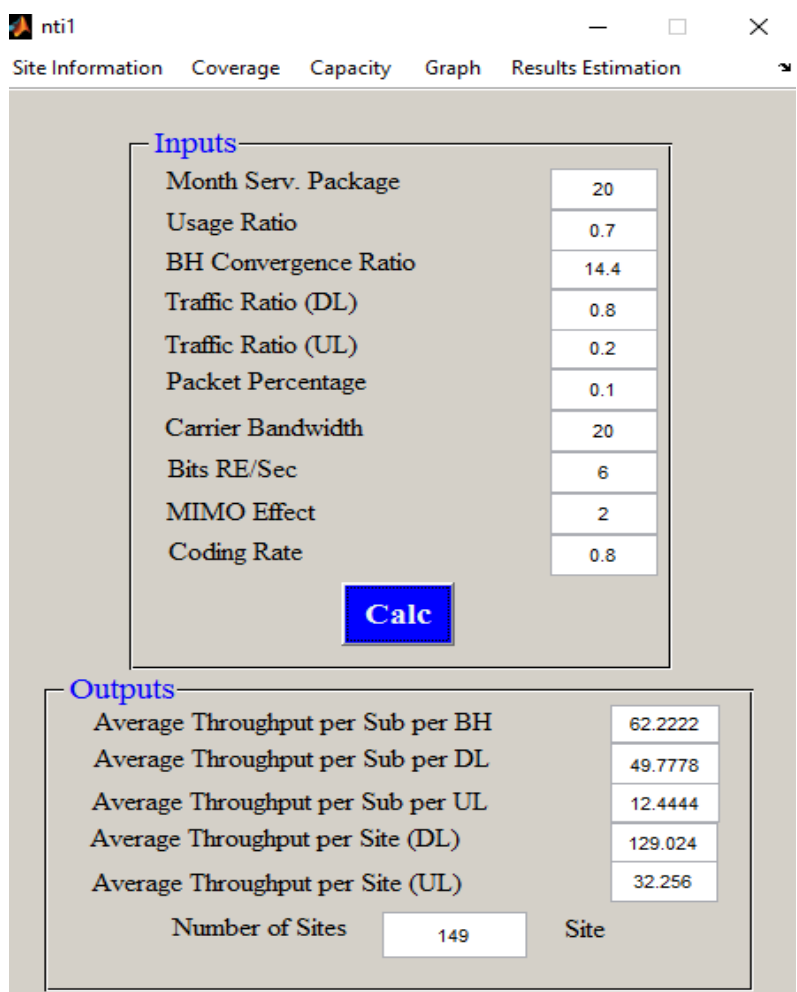

Fig. 4 GUI capacity calculations. 


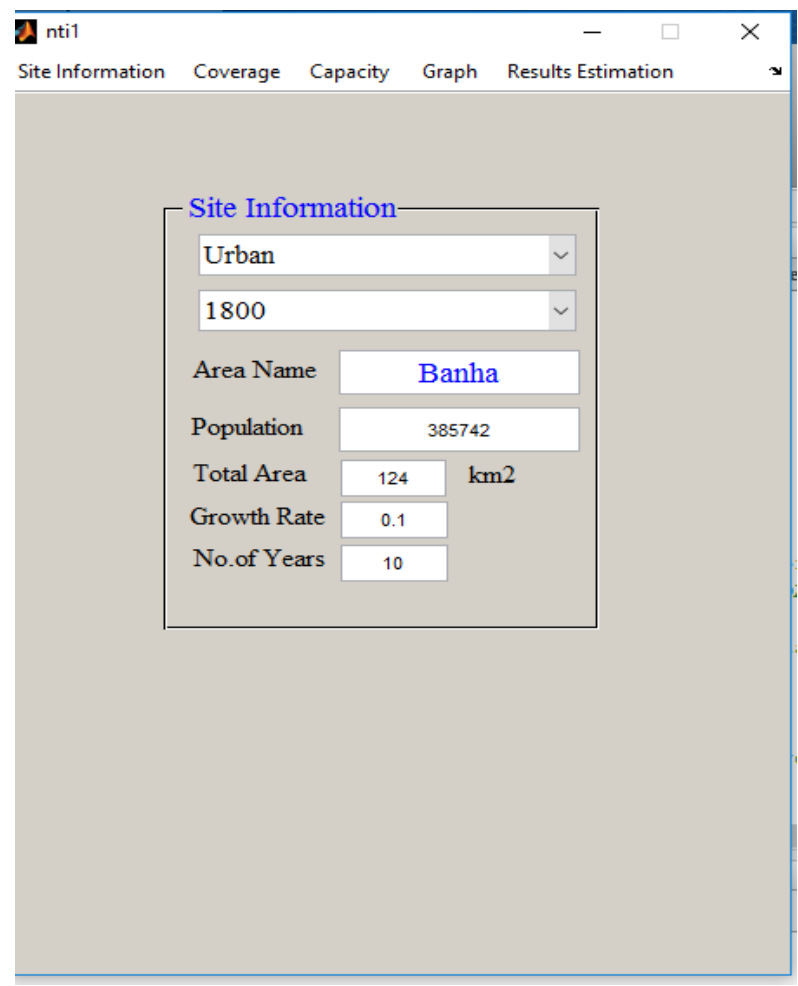

Fig. 5 GUI site information calculations.

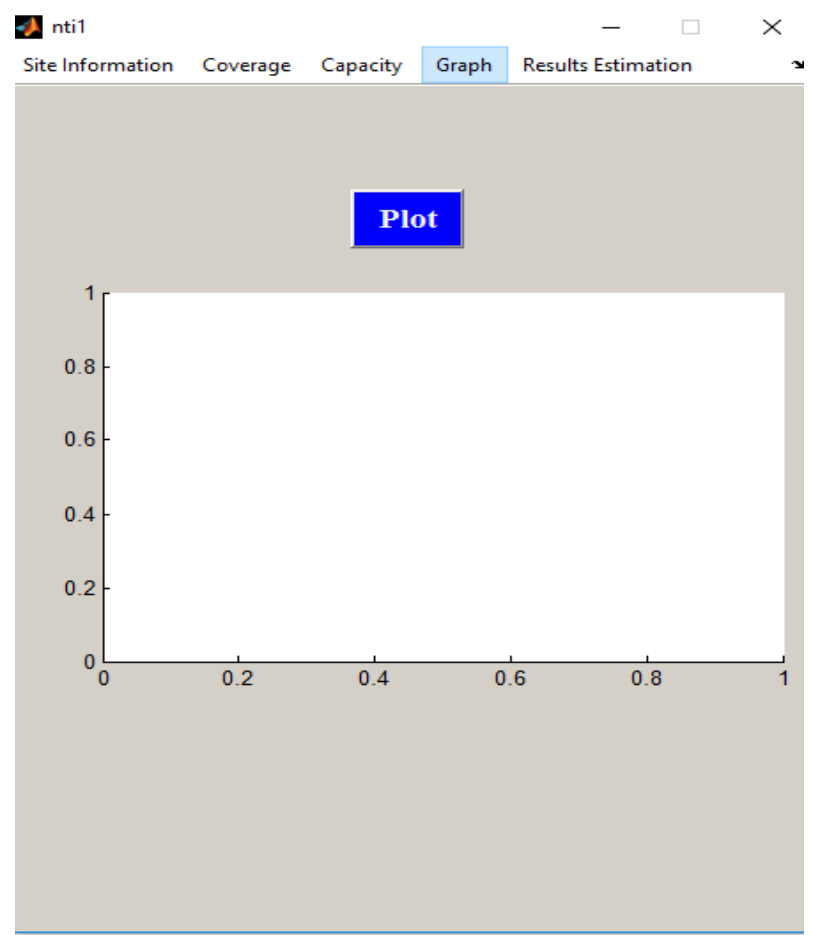

Fig. 6 GUI graphs.

\section{Results Analysis and Verification}

Below, the resulted graphs have been generated with the same parameters as in certificated simulation tool
(ATOLL simulation) used in Ref. [6] to be verified. Addis-Ababa is a research area in Ref. [6] with 460 $\mathrm{km}^{2}$ divided to urban, sub-urban and dense-urban areas. Each area has its own calculations from coverage and capacity point of view.

We were able to obtain same results as indicated in the number of sites from Figs. 7-9 for different services area types. The previous table shows the brief information on Addis-Ababa.

As shown in Fig. 8 as the total number of sides with coverage estimation for different services area in Addis Ababa region.

As shown in Fig. 9 as the total number of sides

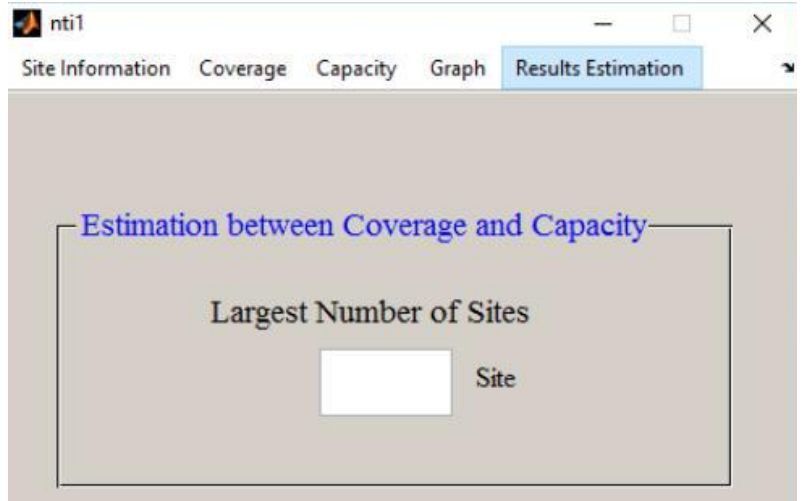

Fig. 7 The number of sites estimated between coverage and capacity.

Table 1 Typical values for the model parameters.

\begin{tabular}{llll}
\hline & Urban & Sub-urban & Dense-urban \\
\hline Area $\left(\mathrm{km}^{2}\right)$ & 170.274 & 220.28 & 70.455 \\
Population & 400,000 & 299,700 & 400,950 \\
Coverage & 255 & 118 & 201 \\
Capacity & 172 & 149 & 199 \\
Cell radius $(\mathrm{km})$ & 0.586 & 0.98 & 0.425 \\
\hline
\end{tabular}

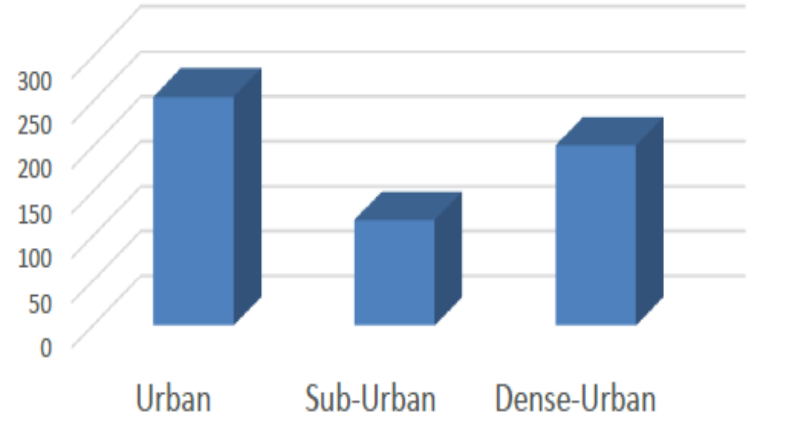

Fig. 8 The total number of sides with coverage estimation for different services area as in Addis Ababa region. 


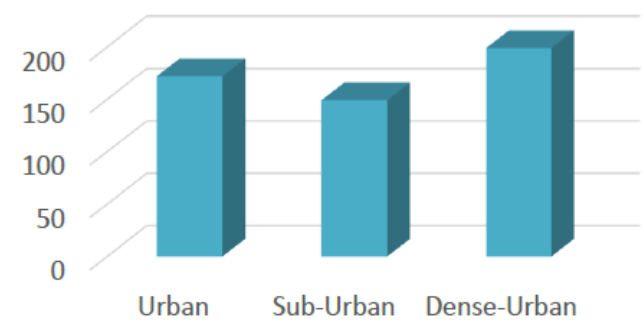

Fig. 9 The total number of sides with capacity estimation for different services area as in Addis Ababa region.

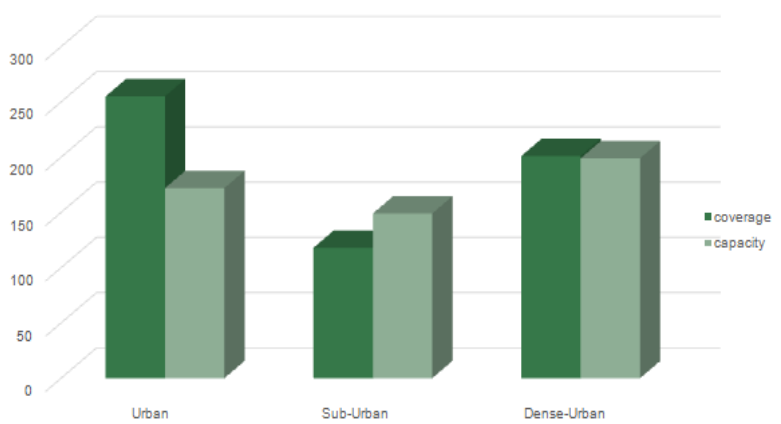

Fig. 10 The total number of sides with coverage and capacity estimation for different services area as in Addis Ababa region.

with capacity estimation for different services area in Addis Ababa region.

As shown in Fig. 10 as the total number of sides with coverage and capacity estimation for different services area in Ref. [6].

\section{Conclusion and Future Works}

This paper is design an effective dimensioning tool set out to plan LTE network using macro cell and this tool is designed to keep the interface simple and to set the functional parts clearly distinguishable.

This paper accomplishes a lot of points, first calculations of link budget using Hata and Cost231 extended Hata propagation models to estimate maximum allowable path loss and use these values to find the number of sites due to coverage. Second, finding the total number of sites due to capacity requirement depends on site throughput in order to support a certain subscriber population, then estimation between coverage and capacity according to maximum number of sites.

This tool is made by GUI of MATLAB. The purpose of designing new tool is to be aware of the concept of planning, steps, different parameters and calculations used to understand planning better. The tool is capable to improve and enhance to support different classes of traffic.

\section{References}

[1] Erik, D., Stefan, P., and Johan, S. 2016. 4G, LTE-Advanced Pro and the Road to 5G. 3rd Edition. London, United Kingdom: Academic Press, Elsevier.

[2] Erik, M., Junaid, A., Amjad, F., and Abad, S. 2010. "Evolution and Development towards 4th Generation Figure 4. The peak throughputs Recent Advances in Image, Audio and Signal Processing ISBN: 978-960-474-350-6 176 (4G) Mobile Communication Systems." Journal of American Science 6 (12).

[3] Lien, S. Y., Chen, K. C., and Lin, Y. 2011. "Toward Ubiquitous Massive Accesses in 3GPP Machine-to-Machine Communications." IEEE Commun. Mag. 49 (Apr.): 66-74.

[4] Saba, S., Ajay, K. G., and Rinku, B. 2012. "Network Simulation Tools Survey." International Journal of Advanced Research in Computer and Communication Engineering 1 (June): 201.

[5] Fitzpatrick, J. M., and Akos, L. 2013. "Computer Programming with MATLAB.” MathWorks.

[6] Gurmu, I. Z., and Negash, Y. 2015. "Long Term Evolution (LTE) Radio Network Dimensioning: Case of Addis Ababa City." M.Sc. in Electrical Engineering, School of Graduate Studies of Addis Ababa University.

[7] 3GPP R2 112863. "Backoff Enhancements for RAN Overload Control." 